Research Article

\title{
Woven Frames in Quaternionic Hilbert Spaces
}

\author{
S. K. Sharma $\mathbb{D},{ }^{1}$ Nitin Sharma $\mathbb{D}^{2}$ and Khole Timothy Poumai ${ }^{3}$ \\ ${ }^{1}$ Department of Mathematics, Kirori Mal College, University of Delhi, Delhi 110 007, India \\ ${ }^{2}$ Department of Mathematics, University of Delhi, Delhi 110 007, India \\ ${ }^{3}$ Department of Mathematics, Motilal Nehru College, University of Delhi, Delhi 110 021, India
}

Correspondence should be addressed to Nitin Sharma; 134nitinsharma@gmail.com

Received 10 December 2020; Revised 9 January 2021; Accepted 21 January 2021; Published 12 February 2021

Academic Editor: Firdous A. Shah

Copyright $(2021$ S. K. Sharma et al. This is an open access article distributed under the Creative Commons Attribution License, which permits unrestricted use, distribution, and reproduction in any medium, provided the original work is properly cited.

In this paper, we introduce and study woven frames in quaternionic Hilbert spaces. We also give some properties of woven frames and give some conditions on family of frames under which it is woven in quaternionic Hilbert spaces. Also, a characterization of weaving frames in terms of a surjective-bounded right linear operator is given.

\section{Introduction and Preliminaries}

In 1952, Duffin and Schaeffer [1] defined frames as follows:

"if $\mathscr{H}$ is a Hilbert space, then $\left\{u_{n}\right\}_{n \in \mathbb{N}} \subset \mathscr{H}$ is said to be a frame for $\mathscr{H}$ if there exist finite constants $c_{l}$ and $c_{u}$ with $0<c_{l} \leq c_{u}$ such that

$$
c_{l}\|u\|^{2} \leq \sum_{n \in \mathbb{N}}\left|\left\langle u, u_{n}\right\rangle\right|^{2} \leq c_{u}\|u\|^{2}, \quad \text { for all } u \in \mathscr{H} . "
$$

The positive constants $c_{l}$ and $c_{u}$ are called lower and upper frame bounds for the frame $\left\{u_{n}\right\}_{n \in \mathbb{N}}$, respectively. Inequality (1) is called the frame inequality for the frame $\left\{u_{n}\right\}_{n \in \mathbb{N}^{*}}$. A frame $\left\{u_{n}\right\}_{n \in \mathbb{N}}$ in $\mathscr{H}$ is said to be

(i) tight: if it is possible to choose $c_{l}=c_{u}$

(ii) Parseval: if it is a tight frame with $c_{l}=c_{u}=1$

Frames have many properties of bases, but lack a very important one, namely, uniqueness. In fact, a frame is an overcomplete sequence of vectors. Members of a frame need not be linearly independent, but still serve as building blocks and are able to recover every vector of the space completely. This property of frames turns out to be very useful particularly in image and signal processing and in communications in general, since this redundancy yields robustness, i.e., less sensitivity to truncation and transmission errors. Frames can be approached in different ways. Two most important ways are to consider frame theory as a branch of functional analysis, which belongs to the branch of pure mathematics, and to consider a class of frames, which is best suited in applications, which lies in the branch of applied mathematics.

Dependencies among the coefficients of the overcomplete representations guarantee a better stability in presence of noise, quantization, and erasures, as well as greater freedom of design comparing to the bases. The redundant counterpart of a basis is called a frame. The role played by redundancy varies with specific applications. One important role is its robustness. That is, by spreading our information over a wider range of vectors, we are more capable to sustain losses and still have accurate reconstruction.

Frame work is an important tool in the study of signal and image processing [2], filter bank theory [3], wireless communications [4], and sigma-delta quantization [5], refer $[4,6-12]$ for more literature, applications, and various generalizations of frames in Hilbert spaces.

1.1. Notations. Throughout this paper, we will denote $\mathfrak{Q}$ to be the noncommutative field of quaternions, $\mathbb{N}$ be the set of natural numbers, and $\mathbb{H}^{R}(\mathfrak{Q})$ be a separable right quaternionic Hilbert space. By the term "right linear operator," we mean a "right $\mathfrak{Q}$-linear operator," and $\mathfrak{B}\left(\mathbb{M}^{R}(\mathfrak{Q})\right)$ denotes the set of all bounded (right $\mathfrak{Q}$-linear) operators of $\mathbb{H}^{R}(\mathfrak{Q})$ and $\mathbb{N}_{m}$ denotes the set of first $m$ natural numbers. 
1.2. Quaternionic Hilbert Spaces. The noncommutative field of quaternions $\mathfrak{Q}$ is a four-dimensional real algebra with unity. In $\mathfrak{Q}, 0$ denotes the null element and 1 denotes the identity with respect to multiplication. It also includes three so-called imaginary units, denoted by $i, j$, and $k$, i.e.,

$$
\mathfrak{Q}=\left\{r_{0}+r_{1} i+r_{2} j+r_{3} k: r_{0}, r_{1}, r_{2}, r_{3} \in \mathbb{R}\right\},
$$

where $i^{2}=j^{2}=k^{2}=-1, i j=-j i=k, j k=-k j=i$, and $k i=-i k=j$. For each $q=r_{0}+r_{1} i+r_{2} j+r_{3} k \in \mathfrak{Q}$, define conjugate of $q$ denoted by $\bar{q}$ as $\bar{q}=r_{0}-r_{1} i-r_{2} j-r_{3} k \in \mathfrak{Q}$. If $q=r_{0}+r_{1} i+r_{2} j+r_{3} k$ is a quaternion, then $r_{0}$ is called the real part of $q$ and $r_{1} i+r_{2} j+r_{3} k$ is called the imaginary part of $q$. The modulus of a quaternion $q=r_{0}+r_{1} i+r_{2} j+r_{3} k$ is defined as

$$
|q|=(\bar{q} q)^{1 / 2}=(q \bar{q})^{1 / 2}=\sqrt{r_{0}^{2}+r_{1}^{2}+r_{2}^{2}+r_{3}^{2}} .
$$

Definition 1. A right quaternionic pre-Hilbert space or right quaternionic inner product space $\mathbb{V}_{R}(\mathfrak{Q})$ is a right quaternionic vector space together with the binary mapping $\langle$.$| . \rangle: \mathbb{V}_{R}(\mathfrak{Q}) \times \mathbb{V}_{R}(\mathfrak{Q}) \longrightarrow \mathfrak{Q}$ (called the Hermitian quaternionic inner product) which satisfies the following properties:
(a) $\overline{\left\langle v_{1} \mid v_{2}\right\rangle}=\left\langle v_{2} \mid v_{1}\right\rangle$ for all $v_{1}, v_{2} \in \mathbb{V}_{R}(\mathfrak{Q})$
(b) $\langle v \mid v\rangle>0$ if $v \neq 0$
(c) $\left\langle v \mid v_{1}+v_{2}\right\rangle=\left\langle v \mid v_{1}\right\rangle+\left\langle v \mid v_{2}\right\rangle$ for all $v, v_{1}, v_{2} \in \mathbb{V}_{R}$ (Q)

(d) $\langle v \mid u q\rangle=\langle v \mid u\rangle q$ for all $v, u \in \mathbb{V}_{R}(\mathfrak{Q})$ and $q \in \mathfrak{Q}$

A right quaternionic inner product space $\mathbb{V}_{R}(\mathfrak{Q})$ also has the following properties:

(i) $\langle v q \mid u\rangle=\bar{q}\langle v \mid u\rangle$ for all $v, u \in \mathbb{V}_{R}(\mathfrak{Q})$ and $q \in \mathfrak{Q}$

(ii) $v_{1} p+v_{2} q \in \mathbb{V}_{R}(\mathfrak{Q})$, for all $v_{1}, v_{2} \in \mathbb{V}_{R}(\mathfrak{Q})$ and $p, q \in \mathfrak{Q}$

Let $\mathbb{V}_{R}(\mathfrak{Q})$ be right quaternionic inner product space with the Hermitian inner product $\langle. \mid$.$\rangle . Define the qua-$ ternionic norm $\|\|:. \mathbb{V}_{R}(\mathfrak{Q}) \longrightarrow \mathbb{R}^{+}$on $\mathbb{V}_{R}(\mathfrak{Q})$ by

$$
\|u\|=\sqrt{\langle u \mid u\rangle}, u \in \mathbb{V}_{R}(\mathfrak{Q}) .
$$

Definition 2. The right quaternionic pre-Hilbert space is called a right quaternionic Hilbert space if it is complete with respect to norm (4) and is denoted by $\mathbb{H}^{R}(\mathfrak{Q})$.

Theorem 1 (the Cauchy-Schwarz inequality, see [13]). If $\mathbb{H}^{R}(\mathfrak{Q})$ is a right quaternionic Hilbert space, then

$$
|\langle u \mid v\rangle|^{2} \leq\langle u \mid u\rangle\langle v \mid v\rangle, \quad \text { for all } u, v \in \mathbb{M}^{R}(\mathfrak{Q}) .
$$

For more literature related to quaternionic Hilbert spaces, one may refer to $[13,14]$.

1.3. Frames in Quaternionic Hilbert Spaces. Khokulan et al. [15] introduced and studied frames for finite-dimensional quaternionic Hilbert spaces, which were further studied in
[16]. Recently, Sharma and Goel [17] introduced and studied frames for separable quaternionic Hilbert spaces. Frames in separable right quaternionic Hilbert spaces $\mathbb{U}^{R}(\mathfrak{Q})$ are defined as follows.

Definition 3 (see [17]). Let $\mathbb{W}^{R}(\mathfrak{Q}$ ) be a right quaternionic Hilbert space and $\left\{u_{i}\right\}_{i \in \mathbb{N}}$ be a sequence in $\mathbb{H}^{R}(\mathfrak{Q})$. Then, $\left\{u_{i}\right\}_{i \in \mathbb{N}}$ is said to be a frame for $\mathbb{W}^{R}(\mathfrak{Q})$ if there exist two finite real constants with $0<r_{1} \leq r_{2}$ such that

$$
r_{1}\|u\|^{2} \leq \sum_{i \in \mathbb{N}}\left|\left\langle u_{i} \mid u\right\rangle\right|^{2} \leq r_{2}\|u\|^{2}, \quad \text { fo all } u \in \mathbb{T}^{R}(\mathfrak{Q})
$$

The positive constants $r_{1}$ and $r_{2}$ are called lower frame and upper frame bounds for the frame $\left\{u_{i}\right\}_{i \in \mathbb{N}}$, respectively. Inequality (6) is called frame inequality for the frame $\left\{u_{i}\right\}_{i \in \mathbb{N}}$. A sequence $\left\{u_{i}\right\}_{i \in \mathbb{N}}$ is called a Bessel sequence for the right quaternionic Hilbert space $\mathbb{M}^{R}(\mathfrak{Q})$ with bound $r_{2}$ if $\left\{u_{i}\right\}_{i \in \mathbb{N}}$ satisfies the right-hand side of inequality (6). A sequence $\left\{u_{i}\right\}_{i \in \mathbb{N}}$ is a tight frame for right quaternionic Hilbert space $\mathbb{M}^{R}(\mathfrak{Q})$ if there exist positive $r_{1}$ and $r_{2}$ satisfying inequality (6) with $r_{1}=r_{2}$, Parseval frame if it is tight with $r_{1}=r_{2}=1$, and exact if it ceases to be a frame in case any one of its element is removed.

If $\left\{u_{i}\right\}_{i \in \mathbb{N}}$ is a frame for $\mathbb{H}^{R}(\mathfrak{Q})$, then the right linear operator $T: \ell_{2}(\mathfrak{Q}) \longrightarrow \mathbb{H}^{R}(\mathfrak{Q})$ defined by

$$
T\left(\left\{q_{i}\right\}_{i \in \mathbb{N}}\right)=\sum_{i \in \mathbb{N}} u_{i} q_{i},\left\{q_{i}\right\} \in \ell_{2}(\mathfrak{Q}),
$$

is called the (right) synthesis operator and the adjoint operator $T^{*}$ is called the (right) analysis operator is given by

$$
T^{*}(u)=\left\{\left\langle u_{i} \mid u\right\rangle\right\}_{i \in \mathbb{N}}, \quad u \in \mathbb{T}^{R}(\mathfrak{Q}) .
$$

Also, the (right) frame operator $S: \mathbb{H}^{R}(\mathfrak{Q}) \longrightarrow \mathbb{M}^{R}(\mathfrak{Q})$ for the frame $\left\{u_{i}\right\}_{i \in \mathbb{N}}$ is a right linear operator given by

$$
\begin{aligned}
S(u)= & T T^{*}(u)=T\left(\left\{\left\langle u_{i} \mid u\right\rangle\right\}_{i \in \mathbb{N}}\right) \\
& =\sum_{i \in \mathbb{N}} u_{i}\left\langle u_{i} \mid u\right\rangle, u \in \mathbb{T}^{R}(\mathfrak{Q}) .
\end{aligned}
$$

1.4. Woven Frames in Hilbert Spaces. Woven frame for separable Hilbert spaces were introduced and studied by Bemrose et al. [18]. They gave the following definition:

Definition 4 (see [18]). A family of frames $\mathfrak{F}=\left\{\left\{u_{i j}\right\}_{i \in \mathbb{N}}: j \in \mathbb{N}_{m}\right\}$ is said to be woven frame for a Hilbert space $\mathscr{H}$ if there are universal constants $c_{l}$ and $c_{u}$ such that, for every partition $P=\left\{\sigma_{j}\right\}_{j \in \mathbb{N}_{m}}$ of $\mathbb{N}$, the family $\mathfrak{F}_{P}=\left\{u_{i j}\right\}_{i \in \sigma_{j}, j \in \mathbb{N}_{m}}$ is a frame for $\mathscr{H}$ with lower and upper frame bounds $c_{l}$ and $c_{u}$, respectively.

Distributed signal processing is the main motivation behind the concept of woven frames. Furthermore, woven frames have inherent applications in wireless sensor networks which are based upon distributed processing under family of frames. For more literature on woven frame, one may refer to [19-21]. 
1.5. Outline of the Paper. In this paper, we introduce and study woven frames in quaternionic Hilbert spaces. Example is given in support of its existence. We also discuss some properties of woven Bessel family and woven frames. Some conditions under which a family of frames is woven in quaternionic Hilbert spaces are given. Furthermore, a characterization of weaving frames in terms of a surjectivebounded right linear operator is given.

\section{Woven Frames in Quaternionic Hilbert Spaces}

We begin this section with the following definition of woven frames in quaternionic Hilbert spaces.

Definition 5. Let $\mathbb{N}_{m}$ be the set of first $m$ natural numbers, $\mathbb{G}^{R}(\mathfrak{Q})$ be a right quaternionic Hilbert space, and $\mathfrak{F}=\left\{\left\{u_{i j}\right\}_{i \in \mathbb{N}}: j \in \mathbb{N}_{m}\right\}$ be a family of frames for $\mathbb{M}^{R}(\mathfrak{Q})$. Then, $\mathfrak{F}$ is said to be woven if there are universal positive real numbers $r_{1}$ and $r_{2}$ so that, for every partition $P=\left\{\sigma_{j}\right\}_{j \in \mathbb{N}_{m}}$ of $\mathbb{N}$, the family $\mathfrak{\mho}_{P}=\left\{u_{i j}\right\}_{i \in \sigma_{j}, j \in \mathbb{N}_{m}}$ is a frame for $\mathbb{\boxplus}^{R}(\mathfrak{Q})$ with lower and upper frame bounds $r_{1}$ and $r_{2}$, respectively. Each family $\mathfrak{F}_{P}$ is called a weaving. If every weaving is a Bessel sequence, then $\mathfrak{F}$ is called a woven Bessel sequence for $\mathbb{M}^{R}(\mathfrak{Q})$.

Next, we give the following lemma:

Lemma 1. Let $\left\{v_{i}\right\}_{i \in \mathbb{N}}$ be a frame for $\mathbb{M}^{R}(\mathfrak{Q})$ with frame bounds $r_{1}$ and $r_{2}$ and $\left\{q_{i}\right\}_{i \in \mathbb{N}} \subset \mathfrak{Q}$ be a sequence such that $r_{3}<\inf _{i \in \mathbb{N}}\left|q_{i}\right|<\sup _{i \in \mathbb{N}}\left|q_{i}\right|<r_{4}$. Define a family $\mathfrak{F}=\left\{\left\{u_{i j}\right\}_{i \in \mathbb{N}}=\right.$ $\left.\left\{j v_{i} q_{i}\right\}_{i \in \mathbb{N}}: j \in \mathbb{N}_{m}\right\}$. Then, $\mathfrak{F}$ is a woven frame for $\mathbb{M}^{R}(\mathfrak{Q})$.

Proof. For any partition $P=\left\{\sigma_{j}\right\}_{j \in \mathbb{N}_{m}}$ of $\mathbb{N}$, we have

$$
\begin{aligned}
r_{1} r_{3}^{2}\|u\|^{2} & \leq \sum_{i \in \mathbb{N}}\left|\left\langle v_{i} q_{i} \mid u\right\rangle\right|^{2} \\
& \leq \sum_{j \in \mathbb{N}_{m}} \sum_{i \in \sigma_{j}}\left|\left\langle j v_{i} q_{i} \mid u\right\rangle\right|^{2} \\
& \leq \sum_{j \in \mathbb{N}_{m}} \sum_{i \in \sigma_{j}}\left|\left\langle u_{i j} \mid u\right\rangle\right|^{2} \\
& \leq m^{2} \sum_{j \in \mathbb{N}_{m}} \sum_{i \in \sigma_{j}}\left|\left\langle v_{i} q_{i} \mid u\right\rangle\right|^{2} \\
& \leq m^{2} r_{2} r_{4}^{2}\|u\|^{2}, u \in \mathbb{H}^{R}(\mathfrak{Q}) .
\end{aligned}
$$

Thus, $\mathfrak{F}$ is a woven frame for $\mathbb{U}^{R}(\mathfrak{Q})$ with lower and upper frame bounds $r_{1} r_{3}^{2}$ and $m^{2} r_{2} r_{4}^{2}$, respectively.

Next, we generalize Lemma 1 in the form of the following theorem.

Theorem 2. Let $\mathfrak{F}=\left\{\left\{u_{i j}\right\}_{i \in \mathbb{N}}: j \in \mathbb{N}_{m}\right\}$ be a woven frame for $\mathbb{Q}^{R}(\mathfrak{Q})$ with universal frame bounds $r_{1}$ and $r_{2}$ and $Q=$ $\left\{\left\{q_{i j}\right\}_{i \in \mathbb{N}}: j \in \mathbb{N}_{m}\right\}$ be a family of sequences in $\mathfrak{Q}$ such that $0<r_{3} \leq \inf \left|q_{i j}\right|^{2} \leq \sup \left|q_{i j}\right|^{2} \leq r_{4}, \quad j \in \mathbb{N}_{m}$. Then, $\mathfrak{F} Q=\left\{\left\{u_{i j}\right.\right.$ $\left.\left.q_{i j i \in \mathbb{N}}: j \in \mathbb{N}_{m}\right\}\right\}$ is a woven frame for $\mathbb{M}^{R}(\mathfrak{Q})$.
Proof. For any partition $P=\left\{\sigma_{j}\right\}_{j \in \mathbb{N}_{m}}$ of $\mathbb{N}$, we have

$$
\begin{aligned}
r_{1} r_{3}\|u\|^{2} & \leq r_{3} \sum_{j \in \mathbb{N}_{m}} \sum_{i \in \sigma_{j}}\left|\left\langle u_{i j} \mid u\right\rangle\right|^{2} \\
& \leq \sum_{j \in \mathbb{N}_{m}} \sum_{i \in \sigma_{j}}\left|\left\langle u_{i j} q_{i j} \mid u\right\rangle\right|^{2} \\
& \leq r_{4} \sum_{j \in \mathbb{N}_{m}} \sum_{i \in \sigma_{j}}\left|\left\langle u_{i j} \mid u\right\rangle\right|^{2} \leq r_{2} r_{4}\|u\|^{2}, \quad u \in \mathbb{H}^{R}(\mathfrak{Q}) .
\end{aligned}
$$

Thus, $\mathfrak{F} Q$ is a woven frame for $\mathbb{t}^{R}(\mathfrak{Q})$ with universal lower and upper frame bounds $r_{1} r_{3}$ and $r_{2} r_{4}$, respectively.

Next, we prove that any finite family of Bessel sequences in a quaternionc Hilbert space is a family of woven Bessel family.

Theorem 3. Let $\mathfrak{F}=\left\{\left\{u_{i j}\right\}_{i \in \mathbb{N}}: j \in \mathbb{N}_{m}\right\}$ be a family of Bessel sequences for $\mathbb{W}^{R}(\mathfrak{Q})$ with Bessel bounds $r_{j}, j \in \mathbb{N}_{m}$. Then, $\mathfrak{F}$ is woven Bessel sequence with universal Bessel bound $\sum_{j \in \mathbb{N}_{m}} r_{j}$.

Proof. For any partition $P=\left\{\sigma_{j}\right\}_{j \in \mathbb{N}_{m}}$ of $\mathbb{N}$, we have

$$
\begin{aligned}
\sum_{j \in \mathbb{N}_{m}} \sum_{i \in \sigma_{j}}\left|\left\langle u_{i j} \mid u\right\rangle\right|^{2} & \leq \sum_{j \in \mathbb{N}_{m}} \sum_{i \in \mathbb{N}}\left|\left\langle u_{i j} \mid u\right\rangle\right|^{2} \\
& \leq\left(\sum_{j \in \mathbb{N}_{m}} r_{j}\right)\|u\|^{2}, \quad u \in \mathbb{T}^{R}(\mathfrak{Q}) .
\end{aligned}
$$

Thus, $\mathfrak{F}$ is woven Bessel sequence with universal Bessel bound $\sum_{j \in \mathbb{N}_{m}} r_{j}$. space

Let $P=\left\{\sigma_{j}\right\}_{j \in \mathbb{N}_{m}}$ be any partition of $\mathbb{N}$, and define the

$$
\underset{j \in \mathbb{N}_{m}}{\oplus} \ell_{2}(\mathfrak{Q})_{\sigma_{j}}=\left\{\left\{q_{i j}\right\}_{i \in \sigma_{j}, j \in \mathbb{N}_{m}} \subset \mathfrak{Q}: \sum_{j \in \mathbb{N}_{m}} \sum_{i \in \sigma_{j}}\left|q_{i j}\right|^{2}<\infty\right\} .
$$

Then, $\oplus_{j \in \mathbb{N}_{m}} \ell_{2}(\mathfrak{Q})_{\sigma_{j}}$ is a right quaternionic Hilbert space with the quaternionic inner product:

$$
\left\langle\left\{p_{i j}\right\}_{i \in \sigma_{j}, j \in \mathbb{N}_{m}} \mid\left\{q_{i j}\right\}_{i \in \sigma_{j}, j \in \mathbb{N}_{m}}\right\rangle=\sum_{j \in \mathbb{N}_{m}} \sum_{i \in \sigma_{j}} \overline{p_{i j} q_{i j}}
$$

Let $\mathfrak{F}=\left\{U_{j}=\left\{u_{i j}\right\}_{i \in \mathbb{N}}: j \in \mathbb{N}_{m}\right\}$ be a woven frame for $\mathbb{Q}^{R}(\mathfrak{Q})$, for any partition $P=\left\{\sigma_{j}\right\}_{j \in \mathbb{N}_{m}}$ of $\mathbb{N}$, then $\mathfrak{\mho}_{P}=\left\{u_{i j}\right\}_{i \in \sigma_{j}, j \in \mathbb{N}_{m}}$ is a frame for $\mathbb{\boxplus}^{R}(\mathfrak{Q})$ and the operator $T_{\widetilde{\mho}_{p}}: \oplus_{j \in \mathbb{N}_{m}} \ell_{2}(\mathfrak{Q})_{\sigma_{j}} \longrightarrow \mathbb{Q}^{R}(\mathfrak{Q})$ defined by

$$
T_{\widetilde{\mho}_{P}}\left(\left\{q_{i j}\right\}_{i \in \sigma_{j}, j \in \mathbb{N}_{m}}\right)=\sum_{j \in \mathbb{N}_{m}} \sum_{i \in \sigma_{j}} u_{i j} q_{i j} \in \mathbb{Q}^{R}(\mathfrak{Q}),
$$

is the synthesis operator of frame $\mathfrak{F}_{P}$. The adjoint operator $T_{\widetilde{\mho}_{p}}^{*}: \mathbb{\boxplus}^{R}(\mathfrak{Q}) \longrightarrow \oplus_{j \in \mathbb{N}_{m}} \ell_{2}(\mathfrak{Q})_{\sigma_{j}}$ of $T_{\widetilde{F}_{p}}$ is given by 


$$
T_{\widetilde{\mho}_{P}}^{*}(u)=\left\{\left\langle u_{i j} \mid u\right\rangle\right\}_{i \in \sigma_{j}, j \in \mathbb{N}_{m}}, \quad u \in \mathbb{G}^{R}(\mathfrak{Q}) .
$$

In view of the above discussion, if we consider $D_{\sigma_{j}}$ to be a diagonal matrix such that

$$
d_{i i}= \begin{cases}1, & \text { if } i \in \sigma_{j}, \\ 0, & \text { otherwise, }\end{cases}
$$

then one can observe that

$$
\begin{aligned}
T_{\widetilde{\mho}_{P}}\left(\left\{q_{i j}\right\}_{i \in \sigma_{j}, j \in \mathbb{N}_{m}}\right) & =\sum_{j \in \mathbb{N}_{m}} T_{U_{j}} D_{\sigma_{j}}\left(\left\{q_{i j}\right\}_{i \in \sigma_{j}, j \in \mathbb{N}_{m}}\right), \\
T_{\mathfrak{\mho}_{P}}^{*}(u) & =\sum_{j \in \mathbb{N}_{m}} D_{\sigma_{j}} T_{U_{j}}^{*}(u),
\end{aligned}
$$

where $T_{U_{j}}$ is the synthesis operator of the frame $U_{j}$.

The frame operator $S_{\mathfrak{F}_{P}}: \mathbb{H}^{R}(\mathfrak{Q}) \longrightarrow \mathbb{T}^{R}(\mathfrak{Q})$ of the frame $\mathfrak{F}_{P}$ is given by

$$
\begin{aligned}
S_{\widetilde{\mho}_{P}}(u) & =T_{\widetilde{\mho}_{P}} T_{\mathfrak{F}_{P}}^{*}(u) \\
& =T_{\widetilde{\mho}_{P}}\left(\left\{\left\langle u_{i j} \mid u\right\rangle\right\}_{i \in \sigma_{j}, j \in \mathbb{N}_{m}}\right) \\
& =\sum_{j \in \mathbb{N}_{m}} \sum_{i \in \sigma_{j}} u_{i j}\left\langle u_{i j} \mid u\right\rangle, \quad u \in \mathbb{T}^{R}(\mathfrak{Q}) .
\end{aligned}
$$

One may easily verify that, for any partition $P$, the frame operator $S_{\mathfrak{F}_{P}}$ is positive, self-adjoint, and invertible.

In the next result, we give a characterization of weaving tight frame.

Theorem 4. Let $\mathfrak{F}=\left\{U_{j}=\left\{u_{i j}\right\}_{i \in \mathbb{N}}: j \in \mathbb{N}_{2}\right\}$ be a woven frame for $\mathbb{H}^{R}(\mathfrak{Q})$, where $T_{U_{j}}$ is the synthesis operator of the frame $U_{j}, j \in \mathbb{N}_{2}$. For any partition $P=\left\{\sigma_{1}, \sigma_{2}=\mathbb{N} \sim \sigma_{1}\right\}$, the weaving $\left\{\left\{u_{i 1}\right\}_{i \in \sigma_{1}} \cup\left\{u_{i 2}\right\}_{i \in \sigma_{2}}\right\}$ is a tight frame for $\mathfrak{H}^{R}(\mathfrak{Q})$ with the frame bound $r$ if and only if $\sum_{j \in \mathbb{N}_{2}} T_{U_{j}} D \sigma_{j} T_{U_{j}}^{*}=r \mathscr{I}_{\mathbb{Q}^{R}(\mathfrak{Q})}$, where $T_{U_{j}}$ is the synthesis operator of the frame $U_{j}$ and $D_{\sigma_{j}}$, a diagonal matrix such that $d_{i i}=\left\{\begin{array}{l}1, \text { if } i \in \sigma_{j}, \\ 0, \text { otherwise. }\end{array}\right.$

Proof. For any partition $P=\left\{\sigma_{j}\right\}_{j \in \mathbb{N}_{2}}$ of $\mathbb{N}$, the synthesis operator of the tight frame $U=\left\{\left\{u_{i 1}\right\}_{i \in \sigma_{1}} \cup\left\{u_{i 2}\right\}_{i \in \sigma_{2}}\right\}$ is $\sum_{j \in \mathbb{N}_{2}} T_{U_{j}} D_{j}$. Therefore, the frame operator of $S_{U}$ is

$$
\begin{aligned}
r \mathscr{I}_{\mathbb{\mathbb { T } ^ { R } ( \mathfrak { Q } )}} & =S_{U} \\
& =\left(\sum_{j \in \mathbb{N}_{2}} T_{U_{j}} D_{j}\right)\left(\sum_{j \in \mathbb{N}_{2}} T_{U_{j}} D_{j}\right)^{*} \\
& =\sum_{j \in \mathbb{N}_{2}} T_{U_{j}} D \sigma_{j} T_{U_{j}}^{*} .
\end{aligned}
$$

In the following, we construct a woven frame with the help of a given woven frame.

Theorem 5. Let $\mathfrak{F}=\left\{U_{j}=\left\{u_{i j}\right\}_{i \in \mathbb{N}^{*}}: j \in \mathbb{N}_{2}\right\}$ be a woven frame for $\mathbb{T}^{R}(\mathfrak{Q})$ with universal frame bounds $r_{1}$ and $r_{2}$ and $S_{U_{j}}$ be the frame operators for the frames $U_{j}, j \in \mathbb{N}_{2}$. If, for $j \in \mathbb{N}_{2}, \quad\left\|S_{U_{j}}^{-1}\right\|\left\|S_{U_{1}}-S_{U_{2}}\right\|<r_{1} / r_{2}, \quad$ then $\quad \mathfrak{G}=\left\{\begin{array}{l}S_{U_{j}}^{-1} \quad U_{j}= \\ \text {, }\end{array}\right.$ $\left.\left\{S_{U_{j}}^{-1} u_{i j}\right\}_{i \in \mathbb{N}}: j \in \mathbb{N}_{2}\right\}$ is also a woven frame for $\mathbb{H}^{R}(\mathfrak{Q})$.

Proof. Let $\left\|S_{U_{2}}^{-1}\right\|\left\|S_{U_{1}}-S_{U_{2}}\right\|<r_{1} / r_{2}$. For any partition $P=$ $\left\{\sigma_{j}\right\}_{j \in \mathbb{N}_{2}}$ of $\mathbb{N}$ and $u \in \mathbb{U}^{R^{2}}(\mathfrak{Q})$, we have

$$
\begin{aligned}
\left(\sum_{j \in \mathbb{N}_{2}} \sum_{i \in \sigma_{j}}\left|\left\langle S_{U_{j}}^{-1} u_{i j} \mid u\right\rangle\right|^{2}\right)^{1 / 2} & =\left(\sum_{j \in \mathbb{N}_{2}} \sum_{i \in \sigma_{j}}\left|\left\langle u_{i j} \mid S_{U_{j}}^{-1} u\right\rangle\right|^{2}\right)^{1 / 2} \\
& =\left(\sum_{i \in \sigma_{1}}\left|\left\langle u_{i 1} \mid S_{U_{1}}^{-1} u\right\rangle\right|^{2}+\sum_{i \in \sigma_{2}}\left|\left\langle u_{i 2} \mid S_{U_{1}}^{-1}+\left(S_{U_{2}}^{-1}-S_{U_{1}}^{-1}\right) u\right\rangle\right|^{2}\right)^{1 / 2} \\
& \geq\left(\sum_{i \in \sigma_{1}}\left|\left\langle u_{i 1} \mid S_{U_{1}}^{-1} u\right\rangle\right|^{2}+\sum_{i \in \sigma_{2}}\left|\left\langle u_{i 2} \mid S_{U_{1}}^{-1} u\right\rangle\right|^{2}\right)^{1 / 2}-\left(\sum_{i \in \sigma_{2}}\left|\left\langle u_{i 2} \mid\left(S_{U_{2}}^{-1}-S_{U_{1}}^{-1}\right) u\right\rangle\right|^{2}\right)^{1 / 2} \\
& \geq \sqrt{r_{1}}\left\|S_{U_{1}}^{-1} u\right\|-\left(\sum_{i \in \mathbb{N}}\left|\left\langle u_{i 2} \mid\left(S_{U_{2}}^{-1}-S_{U_{1}}^{-1}\right) u\right\rangle\right|^{2}\right)^{1 / 2} \\
& \geq \sqrt{r_{1}\left\|S_{U_{1}}^{-1} u\right\|-\sqrt{r_{2}}\left\|S_{U_{2}}^{-1}-S_{U_{1}}^{-1}\right\|\|u\|} \\
& \geq\left(\frac{\sqrt{r_{1}}}{\left\|S_{U_{1}}^{-1}\right\|}-\sqrt{r_{2}}\left\|S_{U_{2}}^{-1}-S_{U_{1}}^{-1}\right\|\right)^{\|u\|}
\end{aligned}
$$


Also,

$$
\begin{aligned}
\sum_{j \in \mathbb{N}_{2}} \sum_{i \in \sigma_{j}}\left|\left\langle S_{U_{j}}^{-1} u_{i j} \mid u\right\rangle\right|^{2} & \leq \sum_{j \in \mathbb{N}_{2}} \sum_{i \in \mathbb{N}}\left|\left\langle S_{U_{j}}^{-1} u_{i j} \mid u\right\rangle\right|^{2} \\
& \leq r_{2}\left(\sum_{j \in \mathbb{N}_{2}}\left\|S_{U_{j}}\right\|^{2}\right)\|u\|^{2} .
\end{aligned}
$$

Next, we give a characterization for the existence of the woven frame in terms of bounded surjective right linear operator.

Theorem 6. Let $\mathfrak{F}=\left\{\left\{u_{i j}\right\}_{i \in \mathbb{N}}: j \in \mathbb{N}_{m}\right\}$ be a family of woven frames for $\mathbb{Q}^{R}(\mathfrak{Q})$ with universal frame bounds $r_{1}$ and $r_{2}$ and $T$ be a bounded right linear operator on $\mathbb{H}^{R}(\mathfrak{Q})$. Then, $T \mathfrak{F}=$ $\left\{\left\{T u_{i j i \in \mathbb{N}}: j \in \mathbb{N}_{m}\right\}\right\}$ is a family of woven frames for $\mathbb{M}^{R}(\mathfrak{Q})$ if and only if $T$ is surjective.

Proof. As $T \mathfrak{F}=\left\{\left\{T u_{i j i \in \mathbb{N}}: j \in \mathbb{N}_{m}\right\}\right\}$ is a family of woven frames for $\mathbb{W}^{R}(\mathfrak{Q})$, therefore, for the partition $P=\left\{\sigma_{j}\right\}_{j \in \mathbb{N}_{m}}$,

$$
\sum_{j \in \mathbb{N}_{m}} \sum_{i \in \sigma_{j}} T u_{i j}\left\langle T u_{i j} \mid u\right\rangle=\operatorname{TS}_{P} T^{*}(u), \quad u \in \mathbb{H}^{R}(\mathfrak{Q}),
$$

where $S_{P}$ be the frame operator for the frame $\left\{u_{i j}\right\}_{i \in \sigma_{j}, j \in \mathbb{N}_{m}}$. Therefore, $\mathrm{TS}_{P} T^{*}$ is the frame operator of $\left\{T u_{i j}\right\}_{i \in \sigma_{j}, j \in \mathbb{N}_{m}}$, so $\mathrm{TS}_{P} T^{*}$ is invertible and $T$ is surjective.

Conversely, let $T$ is surjective. Then, $\operatorname{ran} T=\mathbb{H}^{R}(\mathfrak{Q})$ is closed. Therefore, by Theorem 2.19 in [13], $\operatorname{ker} T \oplus \operatorname{ran} T^{*}=\mathbb{H}^{R}(\mathfrak{Q})=\operatorname{ker} T^{*} \oplus \operatorname{ran} T$. Now, we shall show that $T T^{*}$ is bijective.

If $\quad T T^{*} u=0, u \in \mathbb{H}^{R}(\mathfrak{Q}), \quad$ then $\quad T^{*} u \in \operatorname{ker} T \cap$ $\operatorname{ran} T^{*}=\{0\}$. Therefore, $T^{*} u=0$. Now, let $u \in \operatorname{ker} T^{*}=$ $(\operatorname{ran} T)^{\perp}=\left(\mathbb{M}^{R}(\mathfrak{Q})\right)^{\perp}=\{0\}$. This give $u=0$. Thus, $T T^{*}$ is injective.

As $T T^{*}$ is a positive invertible operator on $\mathbb{T}^{R}(\mathfrak{Q})$, therefore,

$$
0 \leq\left(T T^{*}\right)^{-1} \leq\left\|\left(T T^{*}\right)^{-1}\right\| \mathscr{J}_{\mathbb{Q} \mathfrak{\mathbb { N }}}^{R}(\mathfrak{Q}) .
$$

So, we have

$$
T T^{*}-\left\|\left(T T^{*}\right)^{-1}\right\|^{-1} \mathscr{I}_{\mathbb{W}}^{R}(\mathfrak{Q}) \geq 0 .
$$

This gives

$$
\left\|T^{*} u\right\|^{2} \geq\left\|\left(T T^{*}\right)^{-1}\right\|^{-1}\|u\|^{2}, \quad u \in \mathbb{H}^{R}(\mathfrak{Q}) .
$$

Thus, for any partition $P=\left\{\sigma_{j}\right\}_{j \in \mathbb{N}_{m}}$ of $\mathbb{N}$, we have

$$
\begin{aligned}
r_{1}\left\|\left(T T^{*}\right)^{-1}\right\|^{-1}\|u\|^{2} & \leq r_{1}\left\|T^{*} u\right\|^{2} \\
& \leq \sum_{j \in \mathbb{N}_{m}} \sum_{i \in \sigma_{j}}\left|\left\langle u_{i j} \mid T^{*} u\right\rangle\right|^{2} \\
& =\sum_{j \in \mathbb{N}_{m}} \sum_{i \in \sigma_{j}}\left|\left\langle T u_{i j} \mid u\right\rangle\right|^{2} \\
& \leq r_{2}\|T\|^{2}\||u|\|^{2}, \quad u \in \mathbb{H}^{R}(\mathfrak{Q}) .
\end{aligned}
$$

Hence, $T \mathfrak{F}=\left\{\left\{T u_{i j i \in \mathbb{N}}: j \in \mathbb{N}_{m}\right\}\right\}$ is a family of woven frames for $\mathbb{M}^{R}(\mathfrak{Q})$ with universal lower and upper frame bounds $r_{1}\left\|\left(T T^{*}\right)^{-1}\right\|^{-1}$ and $r_{2}\|T\|^{2}$, respectively.

Corollary 1. Let $\mathfrak{F}=\left\{\left\{u_{i j}\right\}_{i \in \mathbb{N}}: j \in \mathbb{N}_{m}\right\}$ be a family of woven frames for $\mathbb{H}^{R}(\mathfrak{Q})$. Then, one of the frame in the family $\mathfrak{F}$ is considered to be Parseval by considering the family $\sqrt{S_{\lambda}} \mathfrak{\mho}$, where $S_{\lambda}$ is the frame operator of the frame $\left\{u_{i \lambda}\right\}_{i \in \mathbb{N}}$, for some $\lambda \in \mathbb{N}_{m}$.

Next, we give a condition under which a subfamily from a woven frame is removed so that remaining family is a woven frame.

Theorem 7. Let $\mathfrak{F}=\left\{\left\{u_{i j}\right\}_{i \in \mathbb{N}}: j \in \mathbb{N}_{m}\right\}$ be a family of woven frames for $\mathbb{U}^{R}(\mathfrak{Q})$ with universal lower and upper frame bounds $r_{1}$ and $r_{2}$, respectively. If $\mathbb{\mathbb { N }}$ and for some $\lambda \in \mathbb{N}_{m}$, there exists positive real constant $r_{3}<r_{1}$ such that

$$
\sum_{i \in \rrbracket}\left|\left\langle u_{i \lambda} \mid u\right\rangle\right|^{2} \leq r_{3}\|u\|^{2}, \quad u \in \mathbb{H}^{R}(\mathfrak{Q}) .
$$

Then, $\mathfrak{F}_{\mathbb{N}, \rrbracket}=\left\{\left\{u_{i j}\right\}_{i \in \mathbb{N}, \rrbracket}: j \in \mathbb{N}_{m}\right\}$ is a family of woven frames for $\mathbb{H}^{R}(\mathfrak{Q})$.

Proof. For any partition $P=\left\{\eta_{j}\right\}_{j \in \mathbb{N}_{m}}$ of $\mathbb{N}, \mathbb{J}$, we have

$$
\begin{aligned}
\sum_{j \in \mathbb{N}_{m}} \sum_{i \in \eta_{j}}\left|\left\langle u_{i j} \mid u\right\rangle\right|^{2} & \leq \sum_{j \in \mathbb{N}_{m}} \sum_{i \in \eta_{j} \cup \rrbracket}\left|\left\langle u_{i j} \mid u\right\rangle\right|^{2} \\
& \leq r_{2}\|u\|^{2}, \quad u \in \mathbb{M}^{R}(\mathfrak{Q}) .
\end{aligned}
$$

Also,

$$
\begin{aligned}
\sum_{j \in \mathbb{N}_{m}} \sum_{i \in \eta_{j}}\left|\left\langle u_{i j} \mid u\right\rangle\right|^{2} & =\left(\sum_{i \in \eta_{\lambda} \cup \rrbracket}\left|\left\langle u_{i \lambda} \mid u\right\rangle\right|^{2}-\sum_{i \in \rrbracket}\left|\left\langle u_{i \lambda} \mid u\right\rangle\right|^{2}\right)+\sum_{j \in \mathbb{N}_{m}} \sum_{i \in \eta_{j}}\left|\left\langle u_{i j} \mid u\right\rangle\right|^{2} \\
& \geq\left(r_{1}-r_{3}\right)\|u\|^{2}, \quad u \in \mathbb{M}^{R}(\mathfrak{Q}) .
\end{aligned}
$$

Hence, $\mathfrak{F}_{\mathbb{N}, \rrbracket}$ is a family of woven frames for $\mathbb{T}^{R}(\mathfrak{Q})$.

In the next result, we give a positive right linear operator with the help of family of frames, whose existence ensures the weaving of frame family.
Theorem 8. Let $\mathfrak{F}=\left\{\left\{u_{i j}\right\}_{i \in \mathbb{N}}: j \in \mathbb{N}_{m}\right\}$ be a family of frames for $\mathbb{M}^{R}(\mathfrak{Q})$ with frame bounds $r_{1 j}$ and $r_{2 j}, j \in \mathbb{N}_{m}$. For

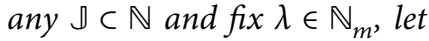


$T_{j}(u)=\sum_{i \in \rrbracket} u_{i j}\left\langle u_{i j} \mid u\right\rangle-\sum_{i \in \rrbracket} u_{i \lambda}\left\langle u_{i \lambda} \mid u\right\rangle, \quad j \in \mathbb{N}_{m}, j \neq \lambda$.

If $T_{j}$ is a positive right linear operator, then $\mathfrak{F}$ is a woven family.
Proof. Let $P=\left\{\eta_{j}\right\}_{j \in \mathbb{N}_{m}}$ be a partition of $\mathbb{N}$. Then, for any $u \in \mathbb{T}^{R}(\mathfrak{Q})$, we have

$$
\begin{aligned}
r_{1 \lambda}\|u\|^{2} & \leq \sum_{i \in \mathbb{N}}\left|\left\langle u_{i \lambda} \mid u\right\rangle\right|^{2} \\
& =\sum_{i \in \eta_{1}}\left|\left\langle u_{i \lambda} \mid u\right\rangle\right|^{2}+\cdots+\sum_{i \in \eta_{j}}\left|\left\langle u_{i \lambda} \mid u\right\rangle\right|^{2}+\cdots+\sum_{i \in \eta_{m}}\left|\left\langle u_{i \lambda} \mid u\right\rangle\right|^{2} \\
& =\sum_{i \in \eta_{1}}\left|\left\langle u_{i \lambda} \mid u\right\rangle\right|^{2}+\cdots+\left\langle u \mid \sum_{i \in \eta_{j}} u_{i \lambda}\left\langle u_{i \lambda} \mid u\right\rangle\right\rangle+\cdots+\sum_{i \in \eta_{m}}\left|\left\langle u_{i \lambda} \mid u\right\rangle\right|^{2} \\
& \leq \sum_{i \in \eta_{1}}\left|\left\langle u_{i \lambda} \mid u\right\rangle\right|^{2}+\cdots+\left\langle u \mid \sum_{i \in \eta_{j}} u_{i j}\left\langle u_{i j} \mid u\right\rangle-T_{j}(u)\right\rangle+\cdots+\sum_{i \in \eta_{m}}\left|\left\langle u_{i \lambda} \mid u\right\rangle\right|^{2} \\
& \leq \sum_{i \in \eta_{1}}\left|\left\langle u_{i \lambda} \mid u\right\rangle\right|^{2}+\cdots+\left\langle u \mid \sum_{i \in \eta_{j}} u_{i j}\left\langle u_{i j} \mid u\right\rangle\right\rangle+\cdots+\sum_{i \in \eta_{m}}\left|\left\langle u_{i \lambda} \mid u\right\rangle\right|^{2} \\
& =\sum_{i \in \eta_{1}}\left|\left\langle u_{i \lambda} \mid u\right\rangle\right|^{2}+\cdots+\sum_{i \in \eta_{j}}\left|\left\langle u_{i j} \mid u\right\rangle\right|^{2}+\cdots+\sum_{i \in \eta_{m}}\left|\left\langle u_{i \lambda} \mid u\right\rangle\right|^{2} \\
& \leq \sum_{i \in \eta_{1}}\left|\left\langle u_{i j} \mid u\right\rangle\right|^{2}+\cdots+\sum_{i \in \eta_{j}}\left|\left\langle u_{i j} \mid u\right\rangle\right|^{2}+\cdots+\sum_{i \in \eta_{m}}\left|\left\langle u_{i j} \mid u\right\rangle\right|^{2} \\
& \leq\left(\sum_{j \in \mathbb{N}_{m}} r_{1 j}\right)\|u\|^{2} .
\end{aligned}
$$

Hence, $\mathfrak{F}$ is a family of woven frames for $\mathbb{U}^{R}(\mathfrak{Q})$ with universal lower and upper frame bounds $r_{1 \lambda}$ and $\left(\sum_{j \in \mathbb{N}_{m}} r_{1 j}\right)$, respectively.

\section{Data Availability}

No data were used to support this study.

\section{Conflicts of Interest}

The authors declare that they have no conflicts of interest.

\section{Acknowledgments}

The research of the second author was sponsored by the University Grants Commission (UGC), vide, Ref. no. 1079/ (OBC) (CSIR-UGC NET DEC. 2016).

\section{References}

[1] R. J. Duffin and A. C. Schaeffer, "A class of nonharmonic Fourier series," Transactions of the American Mathematical Society, vol. 72, no. 2, p. 341, 1952.

[2] R. Balan, P. Casazza, and D. Edidin, "On signal reconstruction without phase," Applied and Computational Harmonic Analysis, vol. 20, no. 3, pp. 345-356, 2006.
[3] H. Bolcskel, F. Hlawatsch, and H. G. Feichtinger, "Frametheoretic analysis of over sampled filter banks," IEEE Transactions on Signal Process, vol. 46, pp. 3256-3268, 1998.

[4] R. W. Heath and A. J. Paulraj, "Linear dispersion codes for MIMO systems based on frame theory," IEEE Transactions on Signal Processing, vol. 50, no. 10, pp. 2429-2441, 2002.

[5] J. J. Lynch, A. M. Powell, and O. Yilmaz, "Sigma-delta quantization and finite frames," IEEE Transactions on Information Theory, vol. 52, no. 5, pp. 1990-2005, 2006.

[6] P. G. Casazza, "The art of frame theory," Taiwanese Journal of Mathematics, vol. 4, no. 2, pp. 129-201, 2000.

[7] P. G. Casazza and G. Kutyniok, "Frames of subspaces," Wavelets, Frames and Operator Theory, vol. 4, 2003.

[8] O. Christensen, "A Paley-Wiener theorem for frames," Proceedings of the American Mathematical Society, vol. 123, no. 7, p. 2199, 1995.

[9] O. Christensen, "An introduction to frames and riesz bases," 2003.

[10] I. Daubechies, A. Grossmann, and Y. Meyer, "Painless nonorthogonal expansions," Journal of Mathematical Physics, vol. 27, no. 5, pp. 1271-1283, 1986.

[11] M. Fornasier, "Quasi-orthogonal decompositions of structured frames," Journal of Mathematical Analysis and Applications, vol. 289, no. 1, pp. 180-199, 2004.

[12] A. Khosravi and K. Musazadeh, "Fusion frames and g-frames," Journal of Mathematical Analysis and Applications, vol. 342, no. 2, pp. 1068-1083, 2008.

[13] R. Ghiloni, V. Moretti, and A. Perotti, "Continuous slice functional calculus in quaternionic Hilbert spaces," IEEE 
Transactions on Signal Processing, vol. 25, no. 83, p. 1350006, 2013.

[14] S. L. Adler, Quaternionic Quantum Mechanics and Quantum Fields, Oxford University Press, New York, NY, USA, 1995.

[15] M. Khokulan, K. Thirulogasanthar, and S. Srisatkunarajah, "Discrete frames on finite dimensional quaternion hilbert spaces," 2008.

[16] S. K. Sharma, "Dual frames on finite dimensional quaternionic Hilbert space," Poincare Journal of Analysis and Applications, vol. 3, no. 2, pp. 79-88, 2016.

[17] S. K. Sharma, S. Goel, and S. Goel, "Frames in quaternionic Hilbert spaces," Zurnal Matematiceskoj Fiziki, Analiza, Geometrii, vol. 15, no. 3, pp. 395-411, 2019.

[18] T. Bemrose, P. G. Casazza, K. Gröchenig, and M. C. Lammers, "Weaving frames," Operators and Matrices, vol. 10, no. 4, pp. 1093-1116, 2016.

[19] D. Li, "On weaving frames in hilbert spaces," 2008.

[20] A. Rahimi, Z. Samadzadeh, and B. Daraby, "Frame-related operators for woven frames," International Journal of Wavelets, Multiresolution and Information Processing, vol. 17, no. 3, 2019.

[21] X. Xiao, Y. Zhu, and L. Găvruţa, "Some properties of K-frames in Hilbert spaces," Results in Mathematics, vol. 63, no. 3-4, pp. 1243-1255, 2013. 